\title{
Improved analysis of ladderane lipids in biomass and sediments using high-performance liquid chromatography/atmospheric pressure chemical ionization tandem mass spectrometry
}

\author{
Ellen C. Hopmans ${ }^{*}$, Michiel V. M. Kienhuis, Jayne E. Rattray, Andrea Jaeschke, \\ Stefan Schouten and Jaap S. Sinninghe Damsté
}

Royal Netherlands Institute for Sea Research, P.O. Box 59, 1790 AB Den Burg, Texel, The Netherlands

Received 29 March 2006; Revised 11 May 2006; Accepted 13 May 2006

\begin{abstract}
Ladderane lipids, containing three or five linearly concatenated cyclobutane moieties, are considered to be unique biomarkers for the process of anaerobic ammonium oxidation, an important link in the oceanic nitrogen cycle. Due to the thermal lability of the strained cyclobutane moieties, the ladderane lipids are difficult to analyze by gas chromatography. A method combining high-performance liquid chromatography coupled to positive ion atmospheric pressure chemical ionization tandem mass spectrometry (HPLC/APCI-MS/MS) was developed for the analysis of the most abundant ladderane lipids, occurring as fatty acids and ether-bound to glycerol. Detection was achieved by selective reaction monitoring of four specific fragmentations per ladderane lipid. Detection limits of $30-35 \mathrm{pg}$ injected on-column and a linear response $\left(r^{2}>0.99\right)$ over nearly 3 orders of magnitude were achieved for all compounds. Using this method, these unique ladderane lipids were for the first time identified in a surface sediment from the Gullmarsfjorden, in concentrations ranging from 1.1-5.5 ng/g for the ladderane fatty acids and of $0.7 \mathrm{ng} / \mathrm{g}$ for the monoether. It is foreseen that this method will allow the investigation of the occurrence of anaerobic ammonium oxidation in natural settings in much greater detail than before. Copyright (C) 2006 John Wiley \& Sons, Ltd.
\end{abstract}

Recently, organisms capable of anaerobic ammonium oxidation ('anammox') in waste waters have been discovered. ${ }^{1}$ These bacteria of the order Planctomycetales combine ammonium and nitrite directly into $\mathrm{N}_{2}$. The anammox process takes place in a special compartment ('anammoxosome') of the cell surrounded by a membrane consisting of unique linearly concatenated cyclobutane (ladderane) lipids (Fig. 1). ${ }^{2}$ These lipids form an extremely dense membrane, which functions as a tight barrier against diffusion. This is required to maintain concentration gradients during the exceptionally slow anammox metabolism and to protect the remainder of the cell from toxic anammox intermediates such as hydrazine. Lipids containing the biochemically unique [5]- and [3]ladderane moieties (Fig. 1, structures A and B, respectively) exist in a variety of forms. In two strains of Candidatus 'Brocadia anammoxidans' they occur, in order of relative abundance, as fatty acids (shown as fatty acid methyl esters (FAMEs) in Fig. 1, structures I-IV), an sn-2-alkyl glycerol monoether (Fig. 1, structure V), alcohols, sn-1,2-dialkyl glycerol diethers, and sn-2-O-alkyl, sn-1-acyl glycerol.

Although initial studies have focused on anammox bacteria from waste water treatment plants, recent findings have indicated that anammox also occurs in the marine environment and may represent a quantitatively important process in the oceanic nitrogen cycle. ${ }^{3-7}$ Kuypers et al. ${ }^{5}$ were the first to identify ladderane lipids in a natural seawater sample using gas chromatography (GC) and GC/mass spectrometry (GC/MS) analysis. Using a combination of molecular biological techniques, ${ }^{15} \mathrm{~N}$-labelling studies and the ladderane lipids as unique biomarkers, they identified the anammox process in the water column of the Black Sea. The pentyl-[3]- and the heptyl-[3]-ladderane fatty acids (II and IV), as well as the monoether (V), were detected (as FAMEs) in concentrations up to $4 \mathrm{ng} / \mathrm{L}$ in the suboxic water zone where the anammox process takes place. Recently, the anammox process was also shown to be important in the Benguela upwelling system. ${ }^{6} \mathrm{Up}$ to $4 \mathrm{ng} / \mathrm{L}$ of the pentyl-[3]and the heptyl-[3]-ladderane fatty acids was detected (as FAMEs) in the oxygen-minimum zone. Although active anammox processes have been shown to occur in sediments using ${ }^{15} \mathrm{~N}$-labelling studies, ${ }^{3,7}$ no reports of ladderanes in sediments have yet been published.

Ladderane lipids are difficult to analyze by GC/MS. ${ }^{8}$ This is due to the thermal lability of the strained cyclobutane 


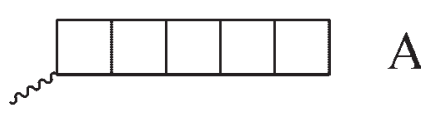

$\mathrm{C}$ COOMe<smiles>[X]CCCCCCCC(=O)OC</smiles><smiles>[X]CCCCCCCCOC(CO)CO</smiles>

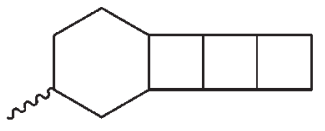

B

\author{
Pentyl-[5]-ladderane FAME $\quad \mathrm{X}=\mathrm{A}$ \\ Pentyl-[3]-ladderane FAME $\quad \mathrm{X}=\mathrm{B}$
}

$\begin{array}{ll}\text { Heptyl-[5]-ladderane FAME } & X=A \\ \text { Heptyl-[3]-ladderane FAME } & X=B\end{array}$

Monoether

$\mathrm{X}=\mathrm{B}$

Figure 1. Structure of ladderane lipids.

moieties making up the [3]- and [5]-ladderane moieties. During GC analysis these components transform into thermally more stable degradation products, which are partially resolved from each other, resulting in the broad peaks observed. To circumvent these problems, a novel method for analysis of the most abundant ladderane lipids, the fatty acids (as FAMEs) and the monoether, using highperformance liquid chromatography coupled to positive ion atmospheric pressure chemical ionization tandem mass spectrometry (HPLC/APCI-MS/MS), was developed. Examples will be shown of ladderane lipid analyses in biomass and sediments.

\section{EXPERIMENTAL}

\section{Standards}

A qualitative standard mixture containing the heptyl-[3]and -[5]-, and pentyl-[5]-ladderane FAMEs (described in Sinninghe Damsté et al. ${ }^{8}$ ) was available for method optimization. Quantitative standards of the fatty acid methyl esters of the heptyl-[3]- and heptyl-[5]-ladderanes, as well as the monoether, were prepared from a large batch of anammox bacterial biomass. ${ }^{2}$ Specifically, freeze-dried culture material was ultrasonically extracted three times each with methanol $(\mathrm{MeOH}), \mathrm{MeOH} /$ dichloromethane (DCM) (1:1, v/v) and DCM. The combined extracts were dried by rotary evaporation. The resulting extract was methylated with $\mathrm{BF}_{3} / \mathrm{MeOH}$ ( $20 \%$ solution) and the reaction mixture partitioned using bidistilled water and DCM. The DCM layer was dried and the resulting residue was separated over an aluminium oxide column with 3 column volumes of hexane/DCM $(4: 1, \mathrm{v} / \mathrm{v}),(3: 2, \mathrm{v} / \mathrm{v}),(1: 1, \mathrm{v} / \mathrm{v})$ and $\mathrm{MeOH} / \mathrm{DCM}(2: 1, \mathrm{v} / \mathrm{v})$. Ladderane FAMEs eluted in the hexane/DCM (4:1) and (3:2) fractions, while the monoether eluted in the $\mathrm{MeOH} / \mathrm{DCM}$ (2:1) fraction. Aliquots of the resulting lipid fractions were further fractionated with reversed-phase HPLC using an Agilent 1100 LC system (Palo Alto, CA, USA). After injection onto two Zorbax Eclipse
XDB-C 8 columns $(4.6 \times 150 \mathrm{~mm}, 5 \mu \mathrm{m}$, Agilent $)$, coupled in series and maintained at $30^{\circ} \mathrm{C}$, the ladderane lipids were eluted with $\mathrm{MeOH}$ at $0.4 \mathrm{~mL} / \mathrm{min}$ while collecting the eluents in $15 \mathrm{~s}$ fractions. The purity of the resulting isolates was assessed by GC and GC/MS according to Sinninghe Damsté et al., 8 and was at least $90 \%$.

A standard mixture of polyunsaturated FAMEs (PUFA-2 mix; Matreya Inc., Pleasant Gap, PA, USA), consisting mainly of C16:0, C18:0, C18:1, C18:2, C18:3, C20:1, C20:2, C20:3 and C22:4, was used to identify interfering peaks in natural samples.

\section{Samples}

Biomass from an anammox reactor, containing both Candidatus 'Scalindua wagneri' and Candidatus 'Kuenenia stuttgartiensis', was a kind gift from B. Kartal (Department of Microbiology, Radboud University Nijmegen, The Netherlands). A composite sample of surface sediments $(0-0.5 \mathrm{~cm})$ was obtained from Gullmarsfjorden, Sweden, where Engström et al. ${ }^{7}$ showed an active anammox process in the sediment.

\section{Sample preparation}

Biomass material and the sediments were freeze-dried, homogenized and ultrasonically extracted with $\mathrm{MeOH} /$ $\operatorname{DCM}(2: 1, \mathrm{v} / \mathrm{v} ; 5 \times)$. The combined extracts were dried by rotary evaporation and $\mathrm{Na}_{2} \mathrm{SO}_{4}$. In the case of the culture cell material, the extract was methylated with $\mathrm{BF}_{3} / \mathrm{MeOH}(20 \%$ solution) and the reaction mixture partitioned using bidistilled water and DCM. The DCM layer was dried and this total lipid fraction was analyzed for ladderanes.

For sediments, the extract was dissolved in DCM and stirred overnight with copper powder, activated with $1 \mathrm{~N}$ $\mathrm{HCl}$, to remove elemental sulfur. The desulfurized extracts were saponified with $1 \mathrm{~N} \mathrm{KOH}$ (in $\mathrm{MeOH} 96 \%$ ) by refluxing for $1 \mathrm{~h}$. After adding $2 \mathrm{~mL}$ bidistilled water, the reaction mixture was partitioned $3 \times$ with DCM to obtain a neutral fraction. A fatty acid fraction was obtained by acidifying the 
residue with $2 \mathrm{~N} \mathrm{HCl} / \mathrm{MeOH}(1: 1, \mathrm{v} / \mathrm{v})$ and partitioning $3 \times$ with DCM. The neutral and fatty acid fractions were both dried over $\mathrm{Na}_{2} \mathrm{SO}_{4}$. The fatty acid fractions were methylated with diazomethane $\left(\mathrm{CH}_{2} \mathrm{~N}_{2}\right)$. To remove very polar components, aliquots were eluted with ethyl acetate over a small column filled with silica. Polyunsaturated fatty acids were removed by eluting the aliquots with DCM over a small column packed with silica impregnated with $\mathrm{AgNO}_{3}$.

Appropriate sample fractions (total lipid extract, fatty acid fraction, or neutral fraction) were dissolved in acetone, filtered through a $0.45 \mu \mathrm{m}, 4 \mathrm{~mm}$ diameter PTFE filter, and analyzed by HPLC/APCI-MS/MS as described below.

\section{Analytical HPLC/APCI-MS/MS}

Analyses were performed using an Agilent 1100 LC system, consisting of an inline membrane degassing unit, thermostated autoinjector and column compartment, coupled to a Quantum TSQ Ultra EM triple quadrupole mass spectrometer (Thermo Inc., San Jose, CA, USA) equipped with an Ion max source with atmospheric pressure chemical ionization (APCI) probe. Separation was achieved on two Zorbax Eclipse $\mathrm{XDB}^{-\mathrm{C}_{8}}$ columns $(4.6 \times 150 \mathrm{~mm}, 5 \mu \mathrm{m}$, Agilent), coupled in series and maintained at $30^{\circ} \mathrm{C}$. Injection volumes varied from 1 to $20 \mu \mathrm{L}$. Ladderanes were eluted with $\mathrm{MeOH}$ at $0.4 \mathrm{~mL} / \mathrm{min}$ with a total run time of $20 \mathrm{~min}$. Detection was achieved using positive ion APCI and selective reaction monitoring (SRM). The following optimal source settings were determined using loop injections of a qualitative mixture of the heptyl-[3]- and -[5]- and pentyl-[5]ladderane FAMEs, and monoether: ${ }^{8}$ vaporizer temperature $475^{\circ} \mathrm{C}$, discharge current $2.5 \mu \mathrm{A}$, sheath gas $\left(\mathrm{N}_{2}\right)$ pressure 50 (arbitrary units), auxiliary gas $\left(\mathrm{N}_{2}\right)$ pressure 5 (arbitrary units), capillary temperature $350^{\circ} \mathrm{C}$, source CID $-10 \mathrm{~V}$. The argon pressure was maintained at $1.5 \mathrm{mT}$ Torr in the second quadrupole. Optimal collision energies varied depending on the monitored reaction and were determined by automatic tuning.

The heptyl-[3]- and -[5]-ladderane FAMEs, as well as the monoether, were quantified by comparing the response in the appropriate SRM trace to the response of known amounts of standards (amounts injected on-column were $30 \mathrm{pg}$ to
$14.5 \mathrm{ng}, 35 \mathrm{pg}$ to $17.7 \mathrm{ng}$, and $10 \mathrm{pg}$ to $10.6 \mathrm{ng}$ for the heptyl[3]-ladderane FAME, heptyl-[5]-ladderane FAME, and the monoether, respectively). No quantitative standard was available for the pentyl-[5]-ladderane FAME and this ladderane was quantified by comparing its response with that of the heptyl-[5]-ladderane FAME.

\section{RESULTS AND DISCUSSION}

\section{Development of the MS/MS method}

In order to determine which reactions should be monitored in the SRM protocol, MS/MS experiments at various collision energies were performed for each ladderane lipid. APCIMS/MS spectra of the protonated molecules of the heptyl[5]- and the heptyl-[3]-ladderane FAMEs are shown in Figs. 2(a) and (b), respectively. For the heptyl-[5]-ladderane FAME, a protonated molecule is obtained at $m / z$ 317. Loss of the methoxy and aldehyde functionalities results in a product ion at $m / z 267$. Loss of the alkyl chain results in a characteristic ion for the [5]-ladderane moiety at $\mathrm{m} / \mathrm{z} 161$, which then fragments in a series of secondary and tertiary reactions to produce clusters of product ions at $m / z$ 145/147, $133,119,105,91$, and 79/81. As expected, the relative abundances of the larger product ion (i.e. $m / z 269,163,133$ / 135) decreased with increasing collision energy, while the abundance of the smaller ions (i.e. $m / z$ 107/109, 93/95, 79/81, 67 increased. The APCI-MS/MS spectrum of the protonated molecule of the pentyl-[5]-ladderane FAME ( $\mathrm{m} / \mathrm{z}$ 289) showed the same fragmentation pattern as the heptyl-[5]ladderane FAME, as they both contain the [5]-ladderane moiety. The APCI-MS/MS spectrum of the heptyl-[3]ladderane FAME, with a protonated molecule at $\mathrm{m} / \mathrm{z} 319$, shows similar fragmentations to the heptyl-[5]-ladderane FAME. However, most product ion clusters are offset by $2 \mathrm{Th}$ compared with their equivalent ions in the mass spectrum of the heptyl-[5]-ladderane FAME, due to the presence of a hexyl group instead of two concatenated cyclobutyl groups in this molecule. The mass spectra obtained by APCI-MS/MS for both the heptyl-[3]- and heptyl-[5]-ladderane FAMEs show similar ions to those in spectra generated by GC/MS. ${ }^{8}$
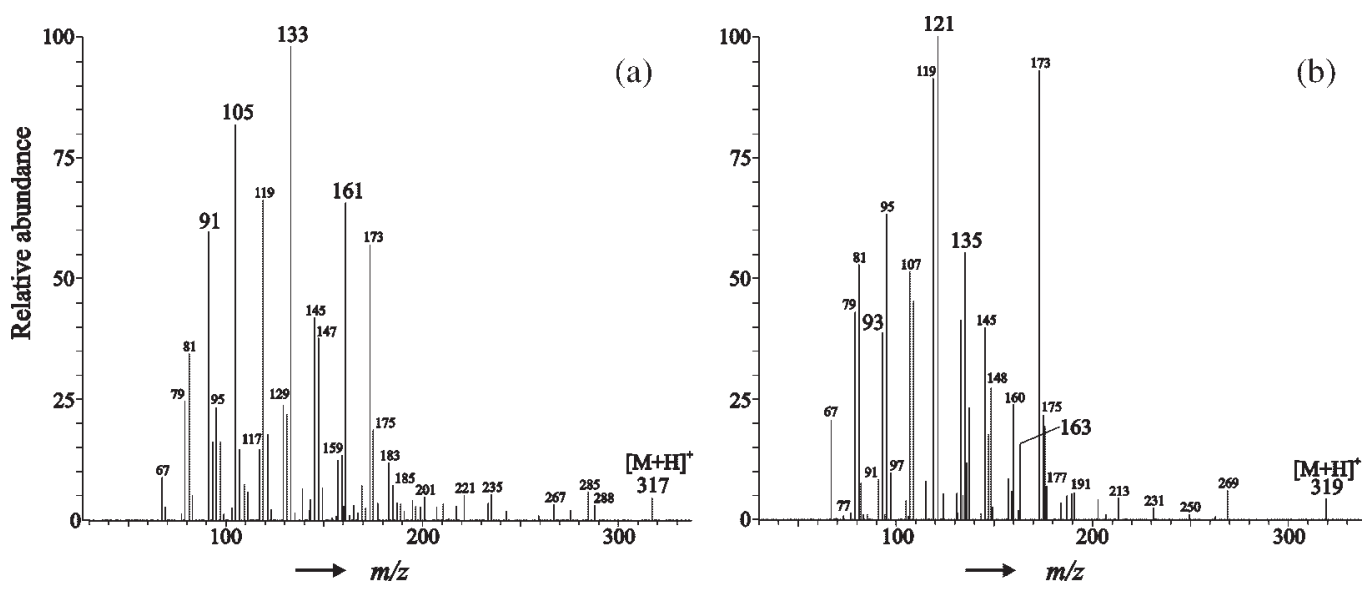

Figure 2. APCI-MS/MS spectra (1.5 mTorr argon, collision energy $-25 \mathrm{~V}$ ) of: (a) protonated molecule $(\mathrm{m} / \mathrm{z} 317)$ of heptyl-[5]-ladderane FAME, and (b) protonated molecule $(\mathrm{m} / \mathrm{z} 319)$ of heptyl-[3]ladderane FAME. Indicated in larger type face are the product ions used for SRM. 
Table 1. Protonated molecules, selected product ions, and respective collision energies for maximal abundance for each monitored ladderane lipid

\begin{tabular}{lcrc}
\hline Ladderane lipid & $\begin{array}{l}{[\mathrm{M}+\mathrm{H}]^{+}} \\
\mathrm{m} / z\end{array}$ & $\begin{array}{l}\text { Product } \\
\mathrm{m} / \mathrm{z}\end{array}$ & $\begin{array}{l}\text { Collision } \\
\text { energy (V) }\end{array}$ \\
\hline Pentyl-[5]-ladderane FAME (I) & 289.3 & 91.1 & 37 \\
& & 105.1 & 33 \\
& & 133.1 & 24 \\
Heptyl-[5]-ladderane FAME (III) & 317.3 & 161.1 & 18 \\
& & 91.1 & 37 \\
& & 105.1 & 35 \\
Heptyl-[3]-ladderane FAME (IV) & \multirow{3}{*}{319.3} & 133.1 & 24 \\
& & 93.1 & 19 \\
& & 121.1 & 24 \\
& & 135.1 & 19 \\
Monoether (V) & 365.3 & 93.1 & 16 \\
& & 121.1 & 29 \\
& & 135.1 & 27 \\
& & 273.2 & 22 \\
\hline
\end{tabular}

The final selection of product ions for SRM was based on their diagnostic value for the concatenated cyclobutane moieties of the ladderane molecules as well as their high relative abundance. Table 1 lists the protonated molecules and selected product ions and respective collision energies for maximal abundance for each monitored ladderane lipid. Identical product ions are monitored for the pentyl- and heptyl-[5]-ladderane FAMEs as they possess identical ladderane moieties. Likewise, three out of four product ions are identical for the heptyl-[3]-ladderane FAME and the
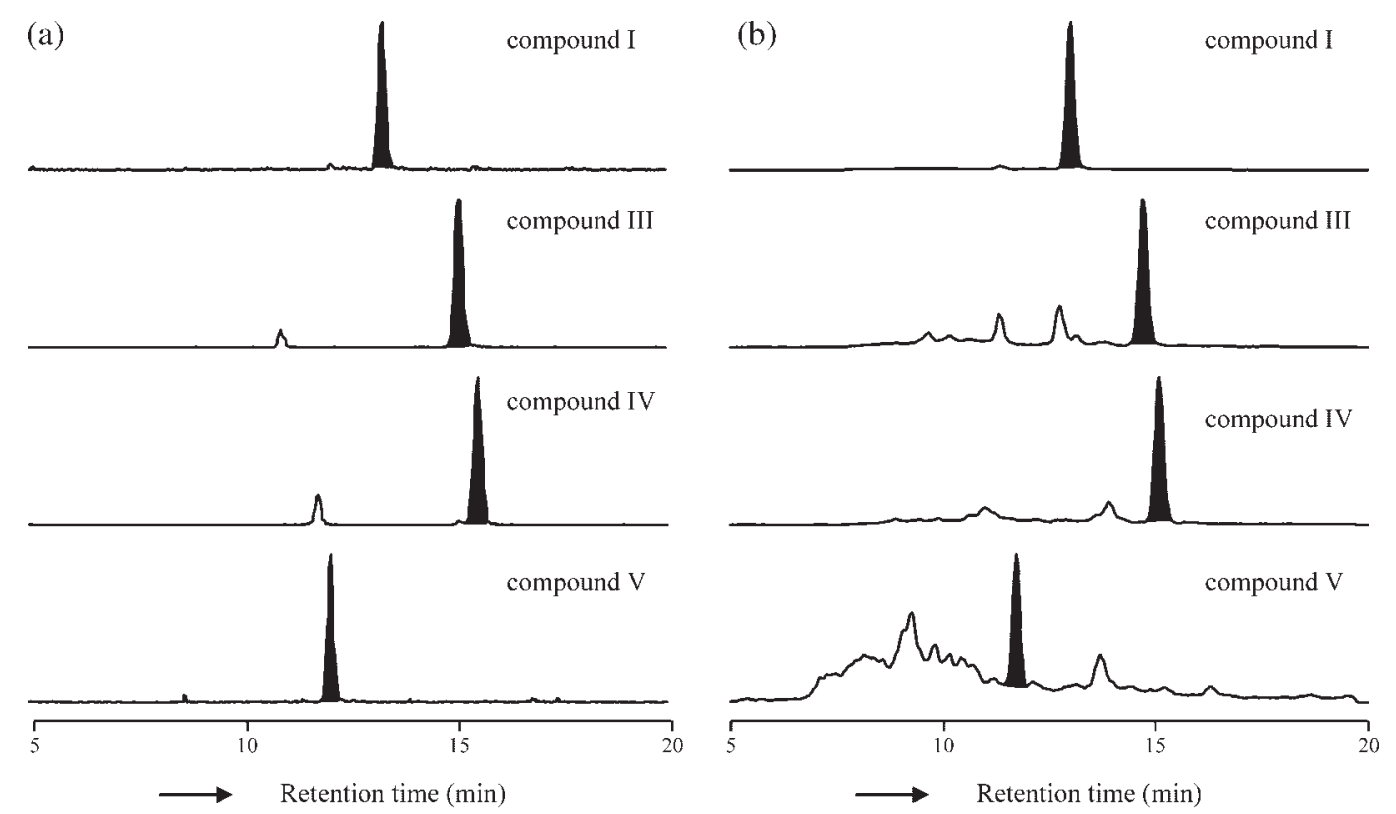

Figure 3. SRM traces of ladderane lipids in: (a) reactor biomass containing both Candidatus 'Scalindua wagneri' and Candidatus 'Kuenenia stuttgartiensis', and (b) surface sediment (0$0.5 \mathrm{~cm}$ ) from Gullmarsfjorden, Sweden. Traces show the pentyl-[5]-, the heptyl-[5]-, the heptyl[3]-ladderane FAMEs, and the monoether (compounds I, III, IV, and V), respectively. For the reactor biomass all traces originate from an analysis of a total lipid extract. For the Gullmarsfjorden sample, the ladderane FAMEs (compounds I, III and IV) were determined in the methylated fatty acid fraction, while the monoether (compound V) was determined in the neutral fraction. glycerol moiety. thermal degradation.

\section{Application of the HPLC/MS/MS method} components.

monoether as they share the [3]-ladderane moiety. The monoether product ion at $m / z 273$ represents the loss of the

External standard curves were prepared for the heptyl-[3]and the heptyl-[5]-ladderane FAMEs, as well as for the monoether. Detection limits, defined at a signal-to-noise ratio of 3 , were 30, 35, and $30 \mathrm{pg}$ injected on-column for the heptyl[3]-ladderane FAME, heptyl-[5]-ladderane FAME, and the monoether, respectively. The response was linear $\left(r^{2}>0.99\right)$ over nearly 3 orders of magnitude for all three compounds. This shows that the developed method is capable of quantitatively and sensitively analyzing ladderane lipids. In comparison with previously used GC and GC/MS methods this method has the benefit of separating these lipids at low column temperatures, thus preventing their

To test the method we first applied it to cell material of anammox bacteria where the presence of ladderane lipids had already been established by GC/MS. Figure 3(a) shows the SRM traces of ladderane lipids as determined in a total extract of reactor biomass containing Candidatus 'Scalindua wagneri' and Candidatus 'Kuenenia stuttgartiensis'. The heptyl-[5]-ladderane FAME was most abundant, followed by the heptyl-[3]-ladderane FAME. Concentrations of the pentyl-[5]-ladderane FAME and the monoether were approximately an order of magnitude less. No major interferences were observed in the SRM traces from other

The method was then tested on a more complex matrix, a surface sediment $(0-0.5 \mathrm{~cm})$ from the Gullmarsfjorden, 
Sweden, where anammox activity had previously been detected using ${ }^{15} \mathrm{~N}$ labelling. ${ }^{7}$ Screening of the fatty acid fraction by GC/MS revealed a large range of saturated and unsaturated FAMEs but ladderane FAMEs could not be detected. Figure 3(b) shows the SRM traces of the ladderane lipids in this same sediment. The FAMEs were determined in the fatty acid fraction, while the monoether was determined in the neutral fraction. After initial analysis of the fatty acid fraction large interfering peaks, eluting just before and coeluting with the target compounds, were observed in all the SRM traces. Analysis of a standard mixture of polyunsaturated FAMEs (PUFAs) showed that these interfering peaks were probably due to the presence of high concentrations of PUFAs in the fatty acid fraction. Although the recorded APCI-MS/MS spectra of PUFAs are distinctly different from those of the ladderane FAMEs (data not shown), the product ions selected for monitoring the ladderane lipids also occur in low abundance in the mass spectra of PUFAs. However, to remove these interferences, the PUFAs can easily be separated from ladderane FAMEs by column chromatography using silica impregnated with $\mathrm{AgNO}_{3}$. PUFAs were effectively removed, while the recoveries of the ladderane FAMEs were over $90 \%$ as tested with standard mixtures. Measured concentrations were 5.5, 1.1, and $1.5 \mathrm{ng} / \mathrm{g}$ sediment for the pentyl-[5]-, heptyl-[5]-, and heptyl-[3]ladderane FAMEs, respectively, and $0.7 \mathrm{ng} / \mathrm{g}$ for the monoether. This is the first report of the unique ladderane lipids in sediments. It is foreseen that this method will allow the investigation of the occurrence of anaerobic ammonium oxidation in natural settings in much greater detail than before.

\section{Acknowledgements}

We gratefully acknowledge B. Kartal and Prof. M. S. M. Jetten of the Department of Micobiology, Radboud University, Nijmegen, for providing biomass from an anammox reactor for this study.

\section{REFERENCES}

1. Strous M, Fuerst JA, Kramer EHM, Logemann S, Muyzer G, van de Pas-Schoonen KT, Webb R, Kuenen JG, Jetten MSM. Nature 1999; 400: 446.

2. Sinninghe Damsté JS, Strous M, Rijpstra WIC, Hopmans EC, Geenevasen JAJ, van Duin ACT, van Niftrik LA, Jetten MSM. Nature 2002; 419: 708.

3. Dalsgaard T, Thamdrup B. Appl. Environ. Microbiol. 2002; 68: 3802.

4. Dalsgaard T, Canfield DE, Petersen J, Thamdrup B, Acuña-González JA. Nature 2003; 422: 606.

5. Kuypers MMM, Sliekers AO, Lavik G, Schmid M, Barker Jørgensen B, Kuenen JG, Sinninghe Damsté JS, Strous M, Jetten MSM. Nature 2003; 422: 608.

6. Kuypers MMM, Lavik G, Woebken D, Schmid M, Fuchs BM, Amann R, Barker Jørgensen B, Jetten MSM. Proc. Natl. Acad. Sci. 2005; 102: 6478.

7. Engström P, Dalsgaard T, Hulth S, Aller RC. Geochim. Cosmochim. Acta 2005; 69: 2057.

8. Sinninghe Damsté JS, Rijpstra WIC, Geenevasen JAJ, Strous M, Jetten MSM. FEBS J. 2005; 272: 4270. 